\title{
UNORIENTED GEOMETRIC FUNCTORS
}

\author{
LAURENCE R. TAYLOR
}

\begin{abstract}
Farrell and Hsiang [2, p. 102] noticed that [5] implies that the geometric surgery groups defined in [6. Chapter 9] do not have the naturality Wall claims for them. Augmenting Wall's definitions using spaces over $\mathbb{R} \mathbb{P}^{\infty}$ and line bundles they fixed the problem.

The definition of geometric Wall groups involves homology with local coefficients and these also lack Wall's claimed naturality.

One would hope that a geometric bordism theory involving nonorientable manifolds would enjoy the same naturality as that enjoyed by homology with local $\mathbb{Z}$ coefficients. A setting for this naturality entirely in terms of local $\mathbb{Z}$ coefficients is presented in this paper.

Applying this theory to the example of non-orientable Wall groups restores much of the elegance of Wall's original approach. Furthermore, a geometric determination of the map induced by conjugation by a group element is given as well as a discussion of further cases beyond the reach of [5].
\end{abstract}

\section{A ReView of LOCAL COEFFicients}

A local coefficient system is a functor from the path groupoid, $\Pi(X)$, of a space $X$ to some category, [4, page 58]. Two coefficient systems are equivalent if there is a natural transformation between the two functors. In the case of interest here the category is the group $\mathbb{Z}$ and such a local coefficient system will be called a $\mathbb{Z}^{t}$-system.

Definition 1.1. For any space $X$, let $\mathcal{Z}^{t}(X)$ denote the category whose objects are $\mathbb{Z}^{t}$-systems on $X$ and whose morphisms are the natural transformations between them.

The data for such a system on a space $X$ can be packaged as a function $\Lambda: \Pi(X) \rightarrow\{ \pm 1\}$ which is a homomorphism of groupoids. One such system is the trivial $\mathbb{Z}^{t}$-system which assigns +1 to every path. A natural transformation, or morphism, or just map, between $\Lambda_{0}$ and $\Lambda_{1}$ is a function $\zeta: X \rightarrow\{ \pm 1\}$ such that for every path $\lambda \in \Pi(X)$,

$$
\Lambda_{0}(\lambda) \cdot \Lambda_{1}(\lambda)=\zeta(\lambda(0)) \cdot \zeta(\lambda(1)) .
$$

Partially supported by the N.S.F.. 
Given any $\mathbb{Z}^{t}$-system $\Lambda$ on $X$ check that for any based loop $L$ in $X$, $\Lambda(L)$ is independent of the base point and defines a homomorphism $H_{1}(X ; \mathbb{Z} / 2 \mathbb{Z}) \rightarrow\{ \pm 1\}$. Define the twist of $\Lambda$ to be the corresponding element $\omega(\Lambda) \in H^{1}(X ; \mathbb{Z} / 2 \mathbb{Z})$. Furthermore, check that two $\mathbb{Z}^{t}$-systems $\Lambda_{0}$ and $\Lambda_{1}$ on $X$ are equivalent if and only if $\omega\left(\Lambda_{0}\right)=\omega\left(\Lambda_{1}\right)$. If $\omega(\Lambda)$ is trivial, $\Lambda$ is said to be untwisted.

Here is a description of all $\mathbb{Z}^{t}$-systems on a space $X$. Pick a set of base points $\left\{b_{i}\right\}$, one in each path component of $X$. For each point $x \in X$ there is a unique $i$ such that $x$ and $b_{i}$ can be joined by a path. For each $x$ pick one path $\lambda_{x}$ joining $x$ to $b_{i}$. Use the constant path to join $b_{i}$ to $b_{i}$. This choice is called a polarization of $\Pi(X)$ and can be characterized as a function $s: X \rightarrow \Pi(X)$ such that $s(x)(0)=x$, $s(x)(1)=b_{i}$ and $s\left(b_{i}\right)$ is constant.

Lemma 1.2. Fix any $\omega \in H^{1}(X ; \mathbb{Z} / 2 \mathbb{Z})$ and any polarization $s$ of $\Pi(X)$. Then any function $f: X \rightarrow\{ \pm 1\}$ such that $f\left(b_{i}\right)=1$ for all $i$ can be uniquely extended to a $\mathbb{Z}^{t}$-system $\Lambda$ such that $\omega(\Lambda)=\omega$ and $f=\Lambda \circ s$.

Proof. Given a path $\lambda$ from $x_{0}$ to $x_{1}$, both points lie in a path component with base point $b_{i}$. There exists a unique $\gamma \in \pi_{1}\left(X, b_{i}\right)$ such that $\gamma$ is equivalent to $s\left(x_{0}\right) \cdot \gamma \cdot s\left(x_{1}\right)^{-1}$. Define $\Lambda(\lambda)=f\left(x_{0}\right) \cdot \omega(\gamma) \cdot f\left(x_{1}\right)$.

Given a subspace $A \subset X$ and a polarization $s_{A}: A \rightarrow \Pi(A)$, construct a polarization $s_{X, s_{A}}: X \rightarrow \Pi(X)$ as follows. Pick base points $\left\{b_{j}\right\} \in X$. If $a_{i} \in A$ is one of the base points in $A$, let $s_{X, s_{A}}\left(a_{i}\right)$ be any path from $a_{i}$ to the appropriate $b_{j}$. If $x \in A$, define $s_{X, s_{A}}(x)=$ $s_{A}(x) \cdot s_{X, s_{A}}\left(a_{i}\right)$ where $x$ is joined in $A$ to $a_{i}$. If $x \in X-A$ then choose any path from $x$ to the appropriate $b_{j}$ using the constant path whenever possible.

Proposition 1.3. Let $A \subset X$ and suppose $\Lambda_{A}$ is a $\mathbb{Z}^{t}$-system on $A$. If there exists a class $\omega \in H^{1}(X ; \mathbb{Z} / 2 \mathbb{Z})$ which restricts to $\omega\left(\Lambda_{A}\right)$, then there exists $\Lambda_{X}$ on $X$ which restricts to $\Lambda_{A}$ and which satisfies $\omega\left(\Lambda_{X}\right)=$ $\omega$.

Proof. Fix a polarization $s_{A}$ of $A$ and a polarization $s_{X, s_{A}}$ of $X$. Define $f: X \rightarrow\{ \pm 1\}$ as follows. If $x \in A$, define $f(x)=\Lambda_{A}\left(s_{A}(x)\right)$ and if $x \in X-A$ let $f(x)=1$. Let $\Lambda_{X}$ be the $\mathbb{Z}^{t}$-system constructed by Lemma 1.2 using $\omega$ for the twist.

Note that if $\zeta$ is a morphism, so is $-\zeta$. Indeed the sign can be switched or not on each path component so the set of morphisms between two $\mathbb{Z}^{t}$-systems on $X$ is an $H^{0}(X ; \mathbb{Z} / 2 \mathbb{Z})$-torsor. An element $\mathfrak{c} \in$ $H^{0}(X ; \mathbb{Z} / 2 \mathbb{Z})$ is equivalent to a homomorphism $H_{0}(X ; \mathbb{Z} / 2 \mathbb{Z}) \rightarrow\{ \pm 1\}$ 
and hence to a function $X \rightarrow\{ \pm 1\}$ which is constant on path components. If $\zeta$ is a morphism, let $\mathfrak{c} \bullet \zeta$ be defined by $\mathfrak{c} \bullet \zeta(x)=\mathfrak{c}(x) \cdot \zeta(x)$ for all $x \in X$.

Proposition 1.4. Let $\iota: A \subset X$ and suppose $\Lambda_{0}$ and $\Lambda_{1}$ are $\mathbb{Z}^{t}$-systems on $X$ with $\omega\left(\Lambda_{0}\right)=\omega\left(\Lambda_{1}\right)$. Suppose $\zeta_{A}: \iota^{*}\left(\Lambda_{0}\right) \rightarrow \iota^{*}\left(\Lambda_{1}\right)$ is given. Then $\zeta_{A}$ extends to $\zeta_{X}: \Lambda_{0} \rightarrow \Lambda_{1}$ provided one of the properties below holds.

(1) $H^{0}(X ; \mathbb{Z} / 2 \mathbb{Z}) \stackrel{\iota^{*}}{\longrightarrow} H^{0}(A ; \mathbb{Z} / 2 \mathbb{Z})$ is onto.

(2) Pick base points $\left\{a_{i}\right\}$ for $A$ and for each path component of $X$ pick a base point $b_{j}$ to be one of the $a_{i}$. Pick paths $\lambda_{i}$ from $a_{i}$ to the appropriate $b_{j}$. For each $i$ require $\Lambda_{0}\left(\lambda_{i}\right) \cdot \Lambda_{1}\left(\lambda_{i}\right)=$ $\zeta_{A}\left(\lambda_{i}(0)\right) \cdot \zeta_{A}\left(\lambda_{i}(1)\right)$.

Proof. Since the twists are the same, there exists $\zeta_{X}^{\prime}: \Lambda_{0} \rightarrow \Lambda_{1}$ which restricts to $\zeta_{A}^{\prime}: \iota^{*}\left(\Lambda_{0}\right) \rightarrow \iota^{*}\left(\Lambda_{1}\right)$. Let $\mathfrak{c}_{A} \in H^{0}(A ; \mathbb{Z} / 2 \mathbb{Z})$ denote the element such that $\zeta_{A}=\mathfrak{c}_{A} \bullet \zeta_{A}^{\prime}$. If (1) holds, pick $\mathfrak{c}_{X} \in H^{0}(X ; \mathbb{Z} / 2 \mathbb{Z})$ which restricts to $\mathfrak{c}_{A}$ and check $\zeta_{X}=\mathfrak{c}_{X} \bullet \zeta_{X}^{\prime}$.

For case (2), pick base points $\left\{b_{j}\right\}$ for $X$ so that each $b_{j}=a_{i_{j}}$. Since $\omega\left(\Lambda_{0}\right)=\omega\left(\Lambda_{1}\right)$ choose an equivalence $\zeta_{X}: \Lambda_{0} \rightarrow \Lambda_{1}$. With a bit of care, further require that $\zeta_{X}\left(a_{i_{j}}\right)=\zeta_{A}\left(a_{i_{j}}\right)$ for all $j$. It follows from the hypotheses that $\left.\zeta_{X}\right|_{A}=\zeta_{A}$.

Proposition 1.5. Let $\Lambda_{0}$ be a $\mathbb{Z}^{t}$-system on $X$ and let $\Lambda_{A}$ be a $\mathbb{Z}^{t}$ system on $A$ where $\iota: A \rightarrow X$ is the inclusion. Let $\zeta: \Lambda_{A} \rightarrow \iota^{*}\left(\Lambda_{0}\right)$ be a map. Then there exists a $\mathbb{Z}^{t}$-system $\Lambda_{1}$ on $X$ such that $\Lambda_{1}$ restricted to $A$ is equal to $\Lambda_{A}$ and $\zeta$ extends to a map $\zeta: \Lambda_{1} \rightarrow \Lambda_{0}$.

Proof. Pick a polarization $s_{X}: X \rightarrow \Pi(X)$ and let $f_{0}: X \rightarrow\{ \pm 1\}$ be $\Lambda_{0} \circ s_{X}$. Define $f_{1}: X \rightarrow\{ \pm 1\}$ by $f_{1}(x)=f_{0}(x) \cdot \zeta(x)$ if $x \in A$ and 1 otherwise. Then $\Lambda_{1}$ is the $\mathbb{Z}^{t}$-system from Lemma 1.2 .

\section{The CATEgory $\mathcal{Z}^{t}($ Top $)$}

A map $f: X_{0} \rightarrow X_{1}$ induces a functor $\Pi\left(X_{0}\right) \rightarrow \Pi\left(X_{1}\right)$ and hence a functor $f^{*}: \mathcal{Z}^{t}\left(X_{1}\right) \rightarrow \mathcal{Z}^{t}\left(X_{0}\right)$. Composition and identity behave correctly, so there is a functor from the category of topological spaces and continuous functions to the category of categories which takes $X$ to the category $\mathcal{Z}^{t}(X)$ and $f$ to the functor $f^{*}$.

Define a category $\mathcal{Z}^{t}(T o p)$ whose objects are all pairs $\left(X, \Lambda_{X}\right)$ for $X$ a topological space and $\Lambda_{X} \in \mathcal{Z}^{t}(X)$. A morphism $\left(X_{0}, \Lambda_{X_{0}}\right) \rightarrow$ $\left(X_{1}, \Lambda_{X_{1}}\right)$ is a pair $(f, \zeta)$ where $f: X_{0} \rightarrow X_{1}$ is any map and $\zeta: \Lambda_{X_{0}} \rightarrow$ $f^{*}\left(\Lambda_{X_{1}}\right)$ is any natural transformation.

Composition is given by $(g, \psi) \circ(f, \zeta)=\left(g \circ f, f^{*}(\psi) \circ \zeta\right)$. The identity for $(X, \Lambda)$ is given by $\left(1_{X}, 1_{\Lambda}\right)$. 
Definition 2.1. The category $\mathcal{Z}^{t}(T o p)$ has a pairing resembling a product. Given $\left(X_{i}, \Lambda_{X_{i}}\right) \in \operatorname{Obj}\left(\mathcal{Z}^{t}(T o p)\right), i=0$, 1 , define a new object

$$
\left(X_{0} \times X_{1}, \Lambda_{X_{0}} \bullet \Lambda_{X_{1}}\right)
$$

Recall $\Pi\left(X_{0} \times X_{1}\right)=\Pi\left(X_{0}\right) \times \Pi\left(X_{1}\right)$ so define

$$
\Lambda_{X_{0}} \bullet \Lambda_{X_{1}}\left(\lambda_{0} \times \lambda_{1}\right)=\Lambda_{X_{0}}\left(\lambda_{0}\right) \cdot \Lambda_{X_{1}}\left(\lambda_{1}\right) .
$$

This construction is not a categorical product since there is no projection onto $X_{0}$ unless $\Lambda_{X_{1}}$ is untwisted. But if $\left(g_{i}, \zeta_{i}\right):\left(Y, \Lambda_{Y}\right) \rightarrow$ $\left(X_{i}, \Lambda_{X_{i}}\right)$ are given, there is a morphism

$$
\left(g_{0} \times g_{1}, \zeta_{0} \bullet \zeta_{1}\right):\left(Y, \Lambda_{Y}\right) \rightarrow\left(X_{0} \times X_{1}, \Lambda_{X_{0}} \bullet \Lambda_{X_{1}}\right)
$$

Definition 2.2. The category $\mathcal{Z}^{t}($ Top) has an involution: it is the identity on objects and sends $(f, \zeta)$ to $(f,-\zeta)$.

Remarks 2.3. Given a bundle over $X$ orient each fibre and associate a $\mathbb{Z}^{t}$-system as follows. Given a path in $X$, use the homotopy lifting property to get a map from the fibre over the initial point to the fibre over the terminal point of the path. Assign +1 to this path if the map is orientation preserving, -1 otherwise. This is a $\mathbb{Z}^{t}$-system and the twist is the first Stiefel-Whitney class of the bundle. There is no preferred choice of orientations if the bundle is non-orientable and even if it is, there are two choices over each path component of $X$. Similar remarks work for spherical fibrations.

\section{Homotopy FunCtors}

The remaining sections describe functors out of subcategories of $\mathcal{Z}^{t}($ Top $)$ or its opposite. Many of these functors have a homotopy invariance which is described next.

Definition 3.1. Let $\mathcal{Z}^{t}(\mathcal{T})$ be a subcategory of $\mathcal{Z}^{t}(T o p)$ and let $\theta_{[0,1]}$ denote the trivial system on $[0,1]$. Say $\mathcal{Z}^{t}(\mathcal{T})$ has homotopies provided the following properties hold.

(1) If $(X, \Lambda) \in \operatorname{Obj}\left(\mathcal{Z}^{t}(\mathcal{T})\right)$ then

$$
\left(X \times[0,1], \Lambda \bullet \theta_{[0,1]}\right) \in \operatorname{Obj}\left(\mathcal{Z}^{t}(\mathcal{T})\right) .
$$

(2) For $i=0$ and $i=1$ the morphisms

$$
\left(\iota_{i}, 1_{\Lambda}\right):(X, \Lambda) \rightarrow\left(X \times[0,1], \Lambda \bullet \theta_{[0,1]}\right)
$$

are in $\mathcal{Z}^{t}(\mathcal{T})$ where $\iota_{i}$ is the evident inclusion. Since $\iota_{i}^{*}\left(\Lambda \bullet \theta_{[0,1]}\right)$ is identical to $\Lambda, 1_{\Lambda}$ is a morphism between the relevant $\mathbb{Z}^{t}$ systems. 
(3) Let $\left(f_{i}, \zeta_{i}\right):\left(X, \Lambda_{X}\right) \rightarrow\left(Y, \Lambda_{Y}\right), i=0,1$ be two maps in $\mathcal{Z}^{t}(\mathcal{T})$ whose $f_{i}$ are homotopic. For any homotopy $F: X \times[0,1] \rightarrow Y$ require the existence of a map $\zeta_{F}: \Lambda_{X} \bullet \theta_{[0,1]} \rightarrow F^{*}\left(\Lambda_{Y}\right)$ such that $\left(F, \zeta_{F}\right)$ is a map in $\mathcal{Z}^{t}(\mathcal{T})$ and the composition $\left(F, \zeta_{F}\right) \circ$ $\left(\iota_{0}, 1_{\zeta_{X}}\right)$ is $\left(f_{0}, \zeta_{0}\right)$.

(4) If $\left(f, \zeta_{i}\right):\left(X, \Lambda_{X}\right) \rightarrow\left(Y, \Lambda_{Y}\right) i=0,1$ are two morphisms with the same $f$, then there exists $\mathfrak{c} \in H^{0}(X ; \mathbb{Z} / 2 \mathbb{Z})$ such that $\zeta_{0}=$ $\mathfrak{c} \bullet \zeta_{1}$. Require that $\left(1_{X}, \mathfrak{c} \bullet 1_{\Lambda_{X}}\right)$ is a map in $\mathcal{Z}^{t}(\mathcal{T})$.

Remarks 3.2. The category $\mathcal{Z}^{t}(T o p)$ has homotopies. The map $\zeta_{F}$ required by (3) is unique. If $r: X \times[0,1] \rightarrow X$ denotes the evident projection, $r$ is a homotopy from $1_{X}$ to itself. The $\mathbb{Z}^{t}$-system $r^{*}(\Lambda)=$ $\Lambda \bullet \theta_{[0,1]}$ so if $\zeta_{r}$ is the identity, $\left(r, 1_{\Lambda \bullet \theta_{[0,1]}}\right) \circ\left(\iota_{i}, 1_{\Lambda}\right)=\left(1_{X}, 1_{\Lambda}\right)$ for both $i=0$ and $i=1$.

Definition 3.3. A functor $\Omega_{*}$ defined on $\mathcal{Z}^{t}(\mathcal{T})$ is a homotopy functor provided $\mathcal{Z}^{t}(\mathcal{T})$ has homotopies and

$$
\Omega_{*}\left(\iota_{0}, 1_{\Lambda}\right) \circ \Omega_{*}\left(r, 1_{\Lambda \bullet \theta_{[0,1]}}\right)=1_{\Omega_{*}\left(X \times[0,1], \Lambda \bullet \theta_{[0,1]}\right)}
$$

There is a similar definition for functors out of $\mathcal{Z}^{t}(\mathcal{T})^{o p}$.

Lemma 3.4. If $\Omega$ is a homotopy functor the two maps

$$
\Omega_{*}\left(\iota_{i}, 1_{\zeta}\right): \Omega_{*}(X, \Lambda) \rightarrow \Omega_{*}\left(X \times[0,1], \Lambda \bullet \theta_{[0,1]}\right)
$$

$i=0,1$ are equal.

Proof. The map $\Omega_{*}\left(r, 1_{\Lambda \bullet \theta_{[0,1]}}\right)$ is an isomorphism inverse to both the maps $\Omega_{*}\left(\iota_{0}, 1_{\Lambda}\right)$ and $\Omega_{*}\left(\iota_{1}, 1_{\Lambda}\right)$. By uniqueness of inverse, $\Omega_{*}\left(\iota_{0}, 1_{\Lambda}\right)=$ $\Omega_{*}\left(\iota_{1}, 1_{\Lambda}\right)$.

Even for a homotopy functor homotopic maps need not induce the same map. To see the problem, let $F: X \times[0,1] \rightarrow Y$ be a homotopy between $f_{i}: X \rightarrow Y$ so $f_{i}=F \circ \iota_{i}$. Let $\zeta_{F}: \Lambda_{X} \bullet \theta_{[0,1]} \rightarrow F^{*}\left(\Lambda_{Y}\right)$ be the map such that $\left(F, \zeta_{F}\right) \circ\left(\iota_{0}, 1_{\Lambda_{X}}\right)$ is $\left(f_{0}, \zeta_{0}\right)$. Then let $\left(F, \zeta_{F}\right) \circ\left(\iota_{1}, 1_{\Lambda_{X}}\right)=$ $\left(f_{1}, \zeta^{\prime}\right)$ so by $3.4 \Omega_{*}\left(f_{0}, \zeta_{0}\right)=\Omega_{*}\left(f_{1}, \zeta^{\prime}\right)$.

The problem is that $\zeta^{\prime}$ may not be $\zeta_{1}$. Since $\zeta^{\prime}$ and $\zeta_{1}$ are maps between the same two $\mathbb{Z}^{t}$-systems, there exists a $\mathfrak{c}_{F} \in H^{0}(X ; \mathbb{Z} / 2 \mathbb{Z})$ such that $\zeta^{\prime}=\mathfrak{c}_{F} \bullet \zeta_{1}$. Then by (41),

$$
\Omega_{*}\left(f_{0}, \zeta_{0}\right)=\Omega_{*}\left(f_{1}, \zeta_{1}\right) \circ \Omega_{*}\left(1_{X}, \mathfrak{c}_{F} \bullet 1_{\Lambda_{X}}\right) .
$$

It remains to identify $\mathfrak{c}_{F}$.

Theorem 3.5. For all $x \in X$

$$
\mathfrak{c}_{F}(x)=\Lambda_{Y}(F(x \times t)) \cdot \zeta_{0}(x) \cdot \zeta_{1}(x)
$$


Proof. By definition $\mathfrak{c}_{F}(x)=\zeta_{1}(x) \cdot \zeta^{\prime}(x)$. Moreover $\zeta^{\prime}(x)=\zeta_{F}(x \times 1)$ and $\zeta_{0}(x)=\zeta_{F}(x \times 0)$. Also by definition,

$$
\zeta_{F}(x \times 0) \cdot \zeta_{F}(x \times 1)=F^{*}\left(\Lambda_{Y}\right)(x \times t) \cdot\left(\Lambda_{X} \bullet \theta_{[0,1]}\right)(x \times t) .
$$

$\operatorname{But}\left(\Lambda_{X} \bullet \theta_{[0,1]}\right)(x \times t)=r^{*}\left(\Lambda_{X}\right)(x \times t)=\Lambda_{X}(r(x \times t))=1$ since $r(x \times t)$ is a constant path. Similarly $F^{*}\left(\Lambda_{Y}\right)(x \times t)=\Lambda_{Y}(F(x \times t))$.

Corollary 3.6. Suppose $X$ is path connected and there exists a point $x \in X$ such that $f_{0}(x)=f_{1}(x)$. Then $F(x \times t)$ is a loop $L$ in $Y$ and $\Lambda_{Y}(F(x \times t))=\omega(L)$.

If $X$ is path connected, then $\mathfrak{c}_{F}= \pm 1$ and $\Omega_{*}\left(1_{X}, \mathfrak{c}_{F} \bullet 1_{\Lambda_{X}}\right)$ is the identity if $\mathfrak{c}_{F}=+1$ and is the map induced by the involution on $\mathcal{Z}^{t}(T o p)$ if $\mathfrak{c}_{F}=-1$ (2.2).

Definition 3.7. Any additive category $\mathscr{A}$ has an involution which is the identity on objects and sends $f$ to $-f$ in the abelian group of morphisms between any two objects. If $\Omega_{*}$ takes values in $\mathscr{A}$, say $\Omega_{*}$ preserves the involution provided $\Omega_{*}(f,-\zeta)=-\Omega_{*}(f, \zeta)$, provided both $(f, \pm \zeta) \in \mathcal{Z}^{t}(\mathcal{T})$. There is a similar definition if $\Omega_{*}$ is a functor out of $\mathcal{Z}^{t}(\mathcal{T})^{o p}$.

\section{TWisted homology AND TWISTED COHOMOLOGY}

There is a detailed treatment of this material in [7, Chapter VI] but here is a quick review. Twisted homology and cohomology come from modifying the singular chain complex $S_{*}(X)$. The chain groups are the same, but the boundary is changed. Recall that $\partial=\sum_{i}(-1)^{i} \partial_{i}$. If $\Lambda$ is a $\mathbb{Z}^{t}$-system, $\partial^{\Lambda}=\sum_{i}(-1)^{i} \partial_{i}^{\Lambda}$ where $\partial_{i}^{\Lambda}$ is defined as follows. Given a singular $r$-simplex $\sigma \rightarrow X, \partial_{i} \sigma$ is a $(r-1)$-simplex. Define $\partial_{i}^{\Lambda} \sigma$ to be $\partial_{i} \sigma$ multiplied by $\Lambda$ applied to the path obtained by applying $\sigma$ to the straight line from the barycenter of $\sigma$ to the barycenter of $\partial_{i} \sigma$. This can be checked to be a chain complex $S_{*}^{\Lambda}(X)$. Homology and cohomology with twisted coefficients is defined as the homology or cohomology of $S_{*}^{\Lambda}$.

Given a natural transformation $\zeta: \Lambda_{0} \rightarrow \Lambda_{1}$, define a chain map $S(\zeta)_{*}: S_{*}^{\Lambda_{0}}(X) \rightarrow S_{*}^{\Lambda_{1}}(X)$ by sending $\sigma$ to $\pm \sigma$ where \pm 1 is the value of the natural transformation applied to the barycenter of $\sigma$. This chain map induces an isomorphism on twisted homology and cohomology groups.

It follows that $S_{*}^{\Lambda}$ and the twisted homology and cohomology groups are functors out of $\mathcal{Z}^{t}(T o p)$ or $\mathcal{Z}^{t}(T o p)^{o p}$. The twisted homology and cohomology are homotopy functors. Furthermore $S_{*}^{\Lambda}, H_{*}$ and $H^{*}$ all preserve involution (2.2). 
The Alexander-Whitney diagonal map induces a chain map

$$
S_{*}^{\Lambda_{X_{0}}}\left(X_{0}\right) \otimes S_{*}^{\Lambda_{X_{1}}}\left(X_{1}\right) \rightarrow S_{*}^{\Lambda_{X_{0}}} \bullet \Lambda_{X_{1}}\left(X_{0} \times X_{1}\right)
$$

so the usual products have twisted versions: given two systems, $\Lambda_{0}$ and $\Lambda_{1}$, there is a bilinear cap product

$$
\cap: H^{r}\left(X ; \mathbb{Z}^{\Lambda_{0}}\right) \otimes H_{r+s}\left(X ; \mathbb{Z}^{\Lambda_{1}}\right) \rightarrow H_{s}\left(X ; \mathbb{Z}^{\Lambda_{0} \bullet \Lambda_{1}}\right)
$$

\section{Poincaré duality SPACES}

A remark that the author has found helpful is that non-orientable manifolds can not be oriented. Without paying enough attention to naturality one can come away with a vague feeling that they can: see the remarks at the end of this section.

If $X$ is path-connected, there can be at most one class of local coefficients $\Lambda$ and one integer $m$ such that $H_{m}\left(X ; \mathbb{Z}^{\Lambda}\right) \cong \mathbb{Z}$ and such that, if $[X]$ is a generator,

$$
\cap[X]: H^{r}\left(X ; \mathbb{Z}^{\Lambda^{\prime}}\right) \rightarrow H_{m-r}\left(X ; \mathbb{Z}^{\Lambda \bullet \Lambda^{\prime}}\right)
$$

is an isomorphism for all $r$ and all $\Lambda^{\prime}$.

If the first Stiefel-Whitney class $w_{1}(M) \in H^{1}(M ; \mathbb{Z} / 2 \mathbb{Z})$ of a connected, closed, compact manifold or Poincaré duality space is used as the twist, (5.1) holds. When $w_{1}(M)=0$ write $H_{*}(M ; \mathbb{Z})$ for homology with trivial $\mathbb{Z}^{t}$-system. The choice of a generator in $H_{m}(M ; \mathbb{Z})$ is often called an orientation and $M$ is said to be oriented. Given two oriented connected, closed, compact manifolds $M_{1}^{m}$ and $M_{2}^{m}$ and a map $f: M_{1} \rightarrow M_{2}, f$ has a degree.

In the non-orientable case the $\mathbb{Z}^{t}$-system is non-trivial and even in the orientable case untwisted but non-trivial systems can appear. Whenever the $\mathbb{Z}^{t}$-system is non-trivial, the class $[M] \in H_{m}\left(M ; \mathbb{Z}^{\Lambda}\right)$ will be called a fundamental class. Given a map $f: M_{0} \rightarrow M_{1}$ which preserves the first Stiefel-Whitney class, there is an induced map

$$
(f, \zeta)_{*}: H_{m}\left(M_{0} ; \mathbb{Z}^{\Lambda_{M_{0}}}\right) \rightarrow H_{m}\left(M_{1} ; \mathbb{Z}^{\Lambda_{M_{1}}}\right)
$$

and $(f, \zeta)_{*}$ has a degree, but if $\zeta$ is replaced by $-\zeta$, the degree switches sign.

Remarks 5.2. The Farrell-Hsiang repair of Wall's problem can be viewed as checking that one can fix a $\mathbb{Z}^{t}$-system over $\mathbb{R P} \mathbb{P}^{\infty}$ and always use the pull-back local system. Whitehead [7, Chapter VI] also discusses naturality using pull-back systems. This results in the following conundrum. Consider the projection $p: S^{2 n} \rightarrow \mathbb{R P}^{2 n}$ and the antipodal map $\alpha$ on $S^{2 n}$ : $\alpha$ commutes with $p$ so there is a commutative triangle of twisted homology groups starting with any $\Lambda$ on $\mathbb{R P}^{2 n}$ with non-trivial 
twist. The induced system on $S^{2 n}$ is untwisted, but not trivial and $\alpha$ ends up having degree +1 . This is all correct but unsettling.

In the approach taken here, one can work with the trivial system on $S^{2 n}$, in which case $\alpha$ has degree -1 but now one must choose an isomorphism between the system induced by $p$ and the trivial one, say $\zeta$. Then the projection maps are $(p, \zeta)$ and $(p,-\zeta), \alpha$ has degree -1 and commutativity still holds. But this shows that $\mathbb{R P}^{2 n}$ can not be "oriented" by orienting $S^{2 n}$ and using " $p_{*}$ " to pick out a fundamental class.

Remarks 5.3. Here is another approach to "orientation". Twisted homology satisfies excision, so $H_{m}\left(M, M-p ; \mathbb{Z}^{\Lambda}\right)=H_{m}\left(D^{m}, S^{m-1} ;\left.\mathbb{Z}^{\Lambda}\right|_{D^{m}}\right)$. Since $D^{m}$ is simply-connected, $H_{m}\left(D^{m}, S^{m-1} ;\left.\mathbb{Z}^{\Lambda}\right|_{D^{m}}\right) \cong \mathbb{Z}$.

There is always a map $H_{m}\left(M ; \mathbb{Z}^{\Lambda}\right) \rightarrow H_{m}\left(M, M-p ; \mathbb{Z}^{\Lambda}\right)$ and if $\omega(\Lambda)=w_{1}(M)$, a fundamental class $[M] \in H_{m}\left(M ; \mathbb{Z}^{\Lambda}\right)$ picks out a generator in $H_{m}\left(D^{m}, S^{m-1} ;\left.\mathbb{Z}^{\Lambda}\right|_{D^{m}}\right)$. Pick the unique morphism from the trivial system to $\left.\mathbb{Z}^{\Lambda}\right|_{D^{m}}$ which is +1 at the point $p$. This gives an orientation on $D^{m}$.

However, whenever $\Lambda$ is not trivial, given any cover of $M$ by disks there will be disks in the cover for which the selected orientation depends on $p$.

\section{Geometric Bordism}

As a warm up exercise, consider unoriented bordism. For the spaces in the category $\mathcal{Z}^{t}(\mathcal{T})$ take $B O \times[0,1]^{k}$ for all integers $k \geqslant 0$. For the $\mathbb{Z}^{t}$-systems take all the ones with non-trivial twist and all their morphisms, and for the maps of spaces take all homotopy equivalences. Fix a $\mathbb{Z}^{t}$-system $\Lambda_{B O}$ on $B O \times[0,1]^{k}$ with non-trivial twist. Hence an object in this "enhanced" bordism theory is a manifold $M$ with a $\mathbb{Z}^{t}$-coefficient system $\Lambda_{M}$, a generator $[M] \in H_{m}\left(M ; \mathbb{Z}^{\Lambda_{M}}\right)$, a map $\nu: M \rightarrow B O \times[0,1]^{k}$ classifying the stable normal bundle and a natural isomorphism $\zeta: \Lambda_{M} \rightarrow \nu^{*}\left(\Lambda_{B O}\right)$.

The definitions for a manifold with boundary present no problems, but manifolds should bound which are not actually equal to a boundary but merely "equivalent" to one. This involves a diffeomorphism of $M$ with an actual boundary and an identification of fundamental classes. Since there is no natural choice of $\zeta$, this requires making $(M, \nu, \mathfrak{c} \bullet[M], \mathfrak{c} \bullet \zeta)$ equal to $(M, \nu,-[M],-\zeta)$. Alternatively one can use Proposition 1.5 to put a $\mathbb{Z}^{t}$-system on $M \times[0,1]$ which alters the sign of $\zeta$ at the two ends.

Bordism defines a homotopy functor on $\mathcal{Z}^{t}(\mathcal{T})$ to abelian groups which preserves orientation. 
Remark 6.1. These definitions can be repeated with $B S O$. Oriented bordism has elements of infinite order, so why are not similar elements present in the unoriented case?

To answer this last question, recall that $B O$ has a homotopy $F: B O \times$ $[0,1] \rightarrow B O$ which is the identity at both ends and such that for each $b \in B O, F(b \times[0,1])$ is a loop which is not null homotopic. Corollary 3.6 implies that the identity map has order 2 so every element in the bordism group has order 2 .

In the oriented case no such homotopy exists.

The case of $\operatorname{Pin}^{ \pm}$structures is interesting from this point of view as well. The bundles are not orientable, but BPin ${ }^{ \pm}=B \operatorname{Spin} \times$ $K(\mathbb{Z} / 2 \mathbb{Z}, 1)$ so there is a homotopy like that for $B O$. However the $\operatorname{Pin}^{ \pm}$bordism groups are not always of exponent 2 . Here the problem is that the homotopy is not a homotopy of lifts over $B O$.

\section{GeOMETRIC SURGERY}

The definition of Wall's geometric surgery groups mimic the unoriented bordism example. Start with a reference $n$-ad $K$ and fix a $\mathbb{Z}^{t}$ coefficient system, $\Lambda_{K}$. An object in the bordism theory is a Poincaré $(n+1)$-ad $X$, a manifold $(n+1)$-ad $M, \mathbb{Z}^{t}$-coefficient systems $\Lambda_{X}$ on $X$ and $\Lambda_{M}$ on $M$, classes $[M] \in H_{m}\left(M ; \mathbb{Z}^{\Lambda_{M}}\right)$ and $[X] \in H_{m}\left(X ; \mathbb{Z}^{\Lambda_{X}}\right)$, maps $g: M \rightarrow X$ and $f: X \rightarrow K$, and finally, isomorphisms $\zeta: \Lambda_{X} \rightarrow$ $f^{*}\left(\Lambda_{K}\right)$ and $\psi: \Lambda_{M} \rightarrow g^{*}\left(\Lambda_{X}\right)$ such that $(g, \psi)_{*}$ has degree 1 . The map $g$ should be covered by a normal map and should have some sort of equivalence on part of the boundary depending on exactly which variant of the Wall group is being constructed.

It is better to denote the resulting bordism groups by $L_{*}\left(K, \Lambda_{K}\right)$ instead of just recording the twist as Wall does. Since the torsion is irrelevant to these discussions it is suppressed.

With these minor alterations in the definition, the material in Wall's Chapter 9 goes through with no difficulty, except for one caveat: the resulting bordism groups are homotopy functors on the full subcategory of $\mathcal{Z}^{t}$ (Top) whose spaces are the homotopy type of CW complexes with a finite 2-skeleton.

Similar results hold for many other variations of surgery theory in the non-orientable case including the $L S$ groups of [6, Chapter 11], the Cappel-Shaneson $\Gamma$ groups [1], and many others. 


\section{SURGERY GROUPS}

The philosophical import of Wall's Chapter 9 is that any "geometric bordism theory" which has a $\pi-\pi$ theorem depends only on the fundamental group.

More explicitly, if $K$ is path-connected, let $u: K \rightarrow K\left(\pi_{1}(K), 1\right)$ be a classifying map for the universal cover. The map $u$ induces an isomorphism

$$
H^{1}\left(K\left(\pi_{1}(K) ; \mathbb{Z} / 2 \mathbb{Z}\right) \rightarrow H^{1}(K ; \mathbb{Z} / 2 \mathbb{Z})\right.
$$

so the set of twists for the two spaces coincide. There is an explicit model for $K(\pi, 1)$ due to Segal [3] denoted $B \pi$ and there is a particular $\mathbb{Z}^{t}$-system $\Lambda_{\omega}$ with twist $\omega \in H^{1}(B \pi ; \mathbb{Z} / 2 \mathbb{Z})$.

The explicit model is Segal's classifying space of a small category where the category is the group in question. There is one 0 -simplex which will be the base point. Each $k$-simplex for $k>0$ is a sequence of $k$ group elements. If $G$ is the group, let $B G$ denote the classifying space. Recall that $B G$ is a $\mathrm{CW}$ complex and it has the homotopy type of a CW complex with finite 2 skeleton if and only if $G$ is finitely generated and finitely presented.

The space $B G$ has an explicit polarization, $s_{G}$, defined as follows. Each point $x \in B G$ lies in the interior of a unique simplex and there is always the straight line path to the initial vertex of this simplex. This gives a path from $x \in B G$ to the base point. Denote the path by $s_{G}(x)$.

Given a twist $\omega \in H^{1}(B G ; \mathbb{Z} / 2 \mathbb{Z})$ define $\Lambda_{\omega}$ to be the $\mathbb{Z}^{t}$-system on $B G$ from Lemma 1.2 with polarization $s_{G}$, function $f: B G \rightarrow\{ \pm 1\}$ identically 1 and twist $\omega$.

If $\psi: H \rightarrow G$ is a group homomorphism then the induced map $B \psi: B H \rightarrow B G$ is piecewise linear on each simplex so

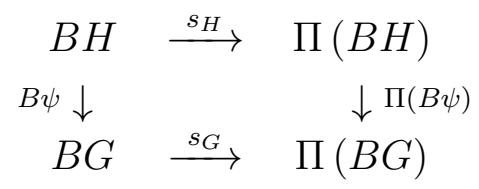

commutes. It follows that $(B \psi)^{*}\left(\Lambda_{\omega}\right)=\Lambda_{B \psi^{*}(\omega)}$ so

$$
\left(B \psi, 1_{\Lambda_{(B \psi)^{*}(\omega)}}\right):\left(B H, \Lambda_{(B \psi)^{*}(\omega)}\right) \rightarrow\left(B G, \Lambda_{\omega}\right) \in \mathcal{Z}^{t}(T o p) .
$$

Since $\Lambda_{(B \psi)^{*}(\omega)}$ is just the identity map, write $\mathbf{B}(\psi, \omega)$ for the map $\left(B \psi, 1_{\Lambda_{(B \psi)^{*}(\omega)}}\right)$. Note that for group homomorphisms, $\psi_{0}: G_{0} \rightarrow G_{1}$ and $\psi_{1}: G_{1} \rightarrow G_{2}, \mathbf{B}\left(\left(\psi_{1} \circ \psi_{0}\right), \omega\right)=\mathbf{B}\left(\psi_{1},\left(B \psi_{0}\right)^{*}(\omega)\right) \circ \mathbf{B}\left(\psi_{0}, \omega\right)$.

Segal's construction has an additional property. Any $g \in G$ gives an automorphism of $G, c_{g}: G \rightarrow G$ defined by $c_{g}(h)=g^{-1} h g$. This inner automorphism is homotopic to the identity since there is a natural 
transformation between the two functors, namely multiplication by $g \in$ $G$. Let $C_{g}: B G \times[0,1] \rightarrow B G$ be the homotopy from $1_{B G}$ at 0 to $c_{g}$ at 1. The homotopy is not base point preserving unless $g$ is the identity: in fact the path $C_{g}(t)$ is just the 1-simplex $g$.

Definition 8.1. A surgery theory is a homotopy functor $\Omega$ on a subcategory $\mathcal{Z}^{t}(\mathcal{T})$ of $\mathcal{Z}^{t}(T o p)$ with the following closure property. Whenever $(K, \Lambda)$ is an object in $\mathcal{Z}^{t}(\mathcal{T})$, then for any $u: K \rightarrow B \pi_{1}(K)$ which induces an isomorphism on $\pi_{1}$ the pair $\left(B \pi_{1}(K), \Lambda_{\left(u^{*}\right)^{-1}(\omega(\Lambda))}\right)$ is in $\mathcal{Z}^{t}(\mathcal{T})$ and for at least one map $\zeta: \Lambda \rightarrow u^{*}\left(\Lambda_{\left(u^{*}\right)^{-1}(\omega(\Lambda))}\right)$ the pair $(u, \zeta)$ is a map in $\mathcal{Z}^{t}(\mathcal{T})$. A surgery theory only depends on $\pi_{1}$ provided

$$
\Omega_{*}(u, \zeta): \Omega_{*}(K, \Lambda) \rightarrow \Omega_{*}\left(B \pi_{1}(K), \Lambda_{\left(u^{*}\right)^{-1}(\omega(\Lambda))}\right)
$$

an isomorphism.

Remark 8.2. Wall's geometric surgery groups are a surgery theory on the full subcategory of $\mathcal{Z}^{t}(T o p)$ whose spaces have the homotopy type of CW complexes with finite 2-skeleton. They preserve the involution. If $*>4$, then the Wall geometric surgery groups only depend on $\pi_{1}$.

Theorem 8.3. Suppose $\Omega_{*}$ is a surgery theory on $\mathcal{Z}^{t}(\mathcal{T})$ and that $G$ is a group such that $\left(B G, \Lambda_{\omega}\right) \in \mathcal{Z}^{t}(\mathcal{T})$. Then

$$
\Omega_{*}\left(\mathbf{B}\left(c_{g}, \omega\right)\right): \Omega_{*}\left(B G, \Lambda_{\omega}\right) \rightarrow \Omega_{*}\left(B G, \Lambda_{\omega}\right)
$$

is the identity if $\omega(g)=+1$ and is $\Omega_{*}\left(1_{B G},-1_{\Lambda_{\omega}}\right)$ if $\omega(g)=-1$. If $\Omega_{*}$ preserves involution, then $\Omega_{*}\left(\mathbf{B}\left(c_{g}, \omega\right)\right)$ is minus the identity if $\omega(g)=$ -1 .

Proof. Apply Theorem 3.5 and Corollary 3.6 note $\zeta_{0}(x)=\zeta_{1}(x)$.

Remark 8.4. Theorem 8.3 is the analogue of the main result in [5] for the geometric Wall groups: it implies that the surgery obstruction map [6, Cor. 9.4.1, p. 90] is natural.

Remark 8.5. This material also implies the geometric version of the conjecture in [5] concerning the map induced by conjugation. Without hypotheses the conjecture is false so some explanation may be helpful. Consider the pair case, $\iota: H \subset G$. Then $B H$ is a subspace of $B G$ and $\iota^{*}\left(\Lambda_{\omega}\right)=\Lambda_{\iota^{*}(\omega)}$. Let $\zeta: \Lambda_{\iota^{*}(\omega)} \rightarrow \iota^{*}\left(\Lambda_{\omega}\right)$ be the identity. The data for the pair should be written $\Omega_{*}\left(\left(B G, \Lambda_{\omega}\right),\left(B H, \Lambda_{\iota^{*}(\omega)}\right), \zeta\right)$.

(1) Any homomorphism of pairs $\psi:\left(G_{0}, H_{0}\right) \rightarrow\left(G_{1}, H_{1}\right)$ induces a map of long exact sequences.

(2) If $g \in H$ then conjugation by $g$ induces an automorphism of the entire long exact sequence of the pair and is multiplication by $\omega(g)$. 
(3) If $g \in G-H$, then $g^{-1} H g$ must be $H$ before $c_{g}$ is even a map of pairs. Fix $\psi: H \rightarrow H$. Then there are examples of groups $G$ with elements $g$ such that $g^{-1} H g=H$ and the induced map on $H$ is $\psi$. Hence nothing can be said in general about the map induced by conjugation on a pair if $g \notin H$.

\section{REFERENCES}

[1] Sylvain E. Cappell and Julius L. Shaneson, The codimension two placement problem and homology equivalent manifolds, Ann. of Math. (2) 99 (1974), 277348. MR 0339216 (49 \#3978)

[2] F. T. Farrell and W. C. Hsiang, Rational L-groups of Bieberbach groups, Comment. Math. Helv. 52 (1977), 89-109. MR 0448372 (56 \#6679)

[3] Graeme Segal, Classifying spaces and spectral sequences, Inst. Hautes Études Sci. Publ. Math. (1968), 105-112. MR 0232393 (38 \#718)

[4] Edwin H. Spanier, Algebraic topology, McGraw-Hill Book Co., New York, 1966. MR 0210112 (35 \#1007)

[5] Laurence R. Taylor, Surgery groups and inner automorphisms (1973), 471-477. Lecture Notes in Math., Vol. 343. MR 0405460 (53 \#9253)

[6] C. T. C. Wall, Surgery on compact manifolds, Academic Press, London, 1970, London Mathematical Society Monographs, No. 1. MR 0431216 (55 \#4217)

[7] George W. Whitehead, Elements of homotopy theory, Graduate Texts in Mathematics, vol. 61, Springer-Verlag, New York, 1978, ISBN 0-387-90336-4. MR 516508 (80b:55001)

E-mail address: taylor.2@nd.edu 